Published in final edited form as:

NEngl J Med. 2011 March 3; 364(9): 797-805. doi:10.1056/NEJMoa1005419.

\title{
Diuretic Strategies in Patients with Acute Decompensated Heart Failure
}

\author{
G. Michael Felker, M.D., M.H.S., \\ Duke University School of Medicine and Duke Heart Center and Duke Clinical Research Institute \\ all in Durham, NC
}

Kerry L. Lee, Ph.D.,

Duke Clinical Research Institute all in Durham, NC

David A. Bull, M.D.,

University of Utah, Salt Lake City

Margaret M. Redfield, M.D.,

Mayo Clinic, Rochester, MN

Lynne W. Stevenson, M.D.,

Brigham and Women's Hospital and Massachusetts General Hospital in Boston

Steven R. Goldsmith, M.D.,

University of Minnesota, Minneapolis

Martin M. LeWinter, M.D.,

University of Vermont, Burlington

Anita Deswal, M.D., M.P.H.,

Baylor College of Medicine and Michael E. DeBakey Veterans Affairs Medical Center, Houston

Jean L. Rouleau, M.D.,

University of Montreal and Montreal Heart Institute, Montreal

Elizabeth O. Ofili, M.D., M.P.H.,

More-house School of Medicine, Atlanta

Kevin J. Anstrom, Ph.D.,

Duke Clinical Research Institute all in Durham, NC

Adrian F. Hernandez, M.D.,

Duke Clinical Research Institute all in Durham, NC

Steven E. McNulty, M.S.,

Duke Clinical Research Institute all in Durham, NC

Eric J. Velazquez, M.D.,

Duke Clinical Research Institute all in Durham, NC

Abdallah G. Kfoury, M.D.,

Copyright $@ 2011$ Massachusetts Medical Society.

Address reprint requests to Dr. Felker at Duke Clinical Research Institute, 2400 Pratt St., Rm. 0311 Terrace Level, Durham, NC

27705, or at michael.felker@duke.edu.

* Clinical sites and principal investigators participating in the National Heart, Lung, and Blood Institute (NHLBI) Heart Failure

Clinical Research Network are listed in the Supplementary Appendix, available at NEJM.org.

No other potential conflict of interest relevant to this article was reported.

Disclosure forms provided by the authors are available with the full text of this article at NEJM.org. 
University of Utah, Salt Lake City

Horng H. Chen, M.B., B.Ch., Mayo Clinic, Rochester, MN

Michael M. Givertz, M.D.,

Brigham and Women's Hospital and Massachusetts General Hospital in Boston

Marc J. Semigran, M.D.,

Massachusetts General Hospital in Boston

Bradley A. Bart, M.D., University of Minnesota, Minneapolis

Alice M. Mascette, M.D., National Heart, Lung, and Blood Institute, Bethesda, MD

Eugene Braunwald, M.D., and

Brigham and Women's Hospital and Massachusetts General Hospital in Boston

Christopher M. O'Connor, M.D.

Duke University School of Medicine and Duke Heart Center and Duke Clinical Research Institute all in Durham, NC

\section{for the NHLBI Heart Failure Clinical Research Network*}

\section{Abstract}

Background-Loop diuretics are an essential component of therapy for patients with acute decompensated heart failure, but there are few prospective data to guide their use.

Methods-In a prospective, double-blind, randomized trial, we assigned 308 patients with acute decompensated heart failure to receive furosemide administered intravenously by means of either a bolus every 12 hours or continuous infusion and at either a low dose (equivalent to the patient's previous oral dose) or a high dose ( 2.5 times the previous oral dose). The protocol allowed specified dose adjustments after 48 hours. The coprimary end points were patients' global assessment of symptoms, quantified as the area under the curve (AUC) of the score on a visualanalogue scale over the course of 72 hours, and the change in the serum creatinine level from baseline to 72 hours.

Results-In the comparison of bolus with continuous infusion, there was no significant difference in patients' global assessment of symptoms (mean AUC, $4236 \pm 1440$ and $4373 \pm 1404$, respectively; $\mathrm{P}=0.47)$ or in the mean change in the creatinine level $(0.05 \pm 0.3 \mathrm{mg}$ per deciliter [4.4 $\pm 26.5 \mu \mathrm{mol}$ per liter] and $0.07 \pm 0.3 \mathrm{mg}$ per deciliter [6.2 $\pm 26.5 \mu \mathrm{mol}$ per liter], respectively; $\mathrm{P}=$ $0.45)$. In the comparison of the high-dose strategy with the low-dose strategy, there was a nonsignificant trend toward greater improvement in patients' global assessment of symptoms in the high-dose group (mean AUC, $4430 \pm 1401$ vs. $4171 \pm 1436$; $\mathrm{P}=0.06$ ). There was no significant difference between these groups in the mean change in the creatinine level $(0.08 \pm 0.3 \mathrm{mg}$ per deciliter [7.1 $\pm 26.5 \mu \mathrm{mol}$ per liter] with the high-dose strategy and $0.04 \pm 0.3 \mathrm{mg}$ per deciliter [3.5 $\pm 26.5 \mu \mathrm{mol}$ per liter] with the low-dose strategy, $\mathrm{P}=0.21$ ). The high-dose strategy was associated with greater diuresis and more favorable outcomes in some secondary measures but also with transient worsening of renal function.

Conclusions-Among patients with acute decompensated heart failure, there were no significant differences in patients' global assessment of symptoms or in the change in renal function when diuretic therapy was administered by bolus as compared with continuous infusion or at a high dose as compared with a low dose. (Funded by the National Heart, Lung, and Blood Institute; ClinicalTrials.gov number, NCT00577135.) 
Acute Decompensated Heart Failure is the most common cause of hospital admissions among patients older than 65 years of age and is responsible for more than 1 million hospitalizations annually in the United States. ${ }^{1}$ Intravenous loop diuretics are an essential component of current treatment and are administered to approximately $90 \%$ of patients who are hospitalized with heart failure. ${ }^{2}$ Despite decades of clinical experience with these agents, prospective data to guide the use of loop diuretics are sparse, and current guidelines are based primarily on expert opinion. ${ }^{3,4}$ As a result, clinical practice varies widely with regard to both the mode of administration and the dosing.

High doses of loop diuretics may have harmful effects, including activation of the reninangiotensin and sympathetic nervous systems, electrolyte disturbances, and worsening of renal function. ${ }^{5}$ In addition, observational studies have shown associations between high doses of diuretics and adverse clinical outcomes, including renal failure, progression of heart failure, and death. ${ }^{6-8}$ Such observations are confounded, however, because high doses of diuretics may be a marker for greater severity of illness rather than a mediator of adverse outcomes.

In addition to uncertainty about dosing, there is uncertainty about the optimal mode of administration. Pharmacokinetic and pharmacodynamic data suggest that there are potential benefits of continuous infusion as compared with intermittent boluses. Although several small studies have evaluated the role of continuous infusion of loop diuretics in patients with heart failure, these studies have been underpowered to address clinical questions. ${ }^{9-16}$

In light of these uncertainties, the National Heart, Lung, and Blood Institute Heart Failure Clinical Research Network conducted the Diuretic Optimization Strategies Evaluation (DOSE) trial, a clinical trial of various diuretic strategies for patients with acute decompensated heart failure.

\section{Methods}

\section{Study Design}

The DOSE study was a prospective, randomized, double-blind, controlled trial..$^{5}$ The study was designed and conducted by the Heart Failure Clinical Research Network (see the Supplementary Appendix, available with the full text of this article at NEJM.org) and was funded entirely by the National Heart, Lung, and Blood Institute. The data coordinating center (Duke Clinical Research Institute) was responsible for data management and statistical analysis. The study protocol, including the statistical analysis plan, is available at NEJM.org. The decision to submit the manuscript for publication was made by the members of the Heart Failure Clinical Research Network Steering Committee, who vouch for the data and the analysis and for the fidelity of the study to the protocol. The study was approved by the institutional review board at each site, and all patients provided written informed consent.

\section{Study Participants}

Patients were eligible for enrollment if they had presented within the previous 24 hours with acute decompensated heart failure, diagnosed on the basis of the presence of at least one symptom (dyspnea, orthopnea, or edema) and one sign (rales, peripheral edema, ascites, or pulmonary vascular congestion on chest radiography) of heart failure. Additional eligibility criteria were a history of chronic heart failure and receipt of an oral loop diuretic for at least 1 month before hospitalization, at a dose between $80 \mathrm{mg}$ and $240 \mathrm{mg}$ daily in the case of furosemide and an equivalent dose in the case of a different loop diuretic $(20 \mathrm{mg}$ of torsemide or $1 \mathrm{mg}$ of bumetanide was considered to be equivalent to $40 \mathrm{mg}$ of furosemide). Thiazide diuretics were permitted if the patient had been taking them on a long-term basis. 
There was no prespecified inclusion criterion with respect to ejection fraction. Patients with systolic blood pressure of less than $90 \mathrm{~mm} \mathrm{Hg}$ or a serum creatinine level that was greater than $3.0 \mathrm{mg}$ per deciliter $(265.2 \mu \mathrm{mol}$ per liter $)$ and patients requiring intravenous vasodilators or inotropic agents (other than digoxin) for heart failure were excluded.

\section{Randomization and Treatment Assignments}

The trial used a 2-by-2 factorial design. Patients were randomly assigned, in a 1:1:1:1 ratio, to either a low-dose strategy (total intravenous furosemide dose equal to their total daily oral loop diuretic dose in furosemide equivalents) or a high-dose strategy (total daily intravenous furosemide dose 2.5 times their total daily oral loop diuretic dose in furosemide equivalents) and to administration of furosemide either by intravenous bolus every 12 hours or by continuous intravenous infusion. Randomization was performed with the use of permuted blocks, stratified according to clinical site. A double-blind, double-dummy design was used so that all patients received both intravenous boluses every 12 hours and a continuous infusion, one of which contained furosemide and the other a saline placebo.

The study treatment, with group assignments concealed, was continued for up to 72 hours. At 48 hours, the treating physician had the option of adjusting the diuretic strategy on the basis of the clinical response. At this time, the physician could increase the dose by 50\% (with the study treatment remaining concealed), maintain the same strategy (with the study treatment remaining concealed), or discontinue intravenous treatment and change to openlabel oral diuretics. After 72 hours, all treatment was open-label at the discretion of the treating physician, who did not have knowledge of the prior study-treatment assignment. An assessment of biomarkers, including creatinine, cystatin $\mathrm{C}$, and $\mathrm{N}$-terminal pro-brain natriuretic peptide, was performed at a central core laboratory at baseline, 72 hours, and 60 days. Patients were followed for clinical events to day 60.

\section{End Points}

The trial had two coprimary end points. The primary efficacy end point was the patient's global assessment of symptoms, measured with the use of a visual-analogue scale and quantified as the area under the curve (AUC) of serial assessments from baseline to 72 hours (see Section 3 in the Supplementary Appendix for a description of the method used for quantification of the area under the curve). ${ }^{17}$ For this assessment, patients were asked to evaluate their general well-being by marking a $10-\mathrm{cm}$ vertical line, with the top labeled "best you have ever felt" and the bottom labeled "worst you have ever felt." We scored the patients' markings on a scale of 0 to 100 by measuring the distance in millimeters from the bottom of the line. The primary safety end point was the change in the serum creatinine level from baseline to 72 hours. See Section 3 in the Supplementary Appendix for more detailed definitions of the study end points.

Prespecified secondary end points included the following: patient-reported dyspnea (as assessed with the use of a visual-analogue scale such as that described above and quantified as the AUC of serial assessments from baseline to 72 hours); changes in body weight and net fluid loss; the proportion of patients who were free from congestion (defined as jugular venous pressure of $<8 \mathrm{~cm}$, with no orthopnea and with trace peripheral edema or no edema) at 72 hours; worsening renal function (defined as an increase in the serum creatinine level of more than $0.3 \mathrm{mg}$ per deciliter) at any time from randomization to 72 hours; worsening or persistent heart failure; treatment failure (see Section 3 in the Supplementary Appendix); changes in biomarker levels at 72 hours, day 7 or discharge, and day 60; and clinical end points, including the composite of death, rehospitalization, or an emergency room visit within 60 days, as well as the composite of total number of days hospitalized or dead during the 60 days after randomization. 


\section{Statistical Analysis}

We estimated that with a sample of 300 patients, the study would have $88 \%$ power to detect a 600-point difference between groups in the AUC of the patients' global assessment score and $88 \%$ power to detect a difference of $0.2 \mathrm{mg}$ per deciliter $(17.7 \mu \mathrm{mol}$ per liter $)$ in the change in the creatinine level between groups, on the basis of estimates of the variability in these outcome measures obtained from previous studies. ${ }^{18-20}$ With respect to the primary efficacy end point, we considered a 600-point difference to be a reasonable estimate of the minimum clinically important difference for this scale (see Section 3 in the Supplementary Appendix).

All analyses were performed according to the intention-to-treat principle. Owing to the use of two coprimary end points (an efficacy and a safety end point), the prespecified threshold for significance for each end point was a $\mathrm{P}$ value of less than 0.025 . For secondary end points, a $\mathrm{P}$ value of less than 0.05 was considered to indicate statistical significance. The treatment groups defined by each treatment factor (mode and dose) were compared with the use of a linear model (for continuous end points), logistic regression (for binary end points), or a Cox model and Kaplan- Meier curves (for time-to-event end points). When differences between two groups that were defined by one of the treatment factors were assessed, the statistical model adjusted for the other factor. In the case of end points for which a relevant baseline value was measured (e.g., serum creatinine level), the analysis was also adjusted for the baseline value of that measure. A test for the presence of an interaction between the two treatment factors was also performed within the statistical framework appropriate for each end point.

\section{Results \\ Patient Population}

A total of 308 patients were enrolled between March 2008 and November 2009 at 26 clinical sites in the United States and Canada (see Section 2 in the Supplementary Appendix). Baseline characteristics for each of the treatment groups are shown in Table 1. The mean age of the patients was 66 years; $27 \%$ were women, and $25 \%$ were black. The patient population had several high-risk features, including a history of hospitalization for heart failure within the previous 12 months (74\% of the patients), moderate renal dysfunction (mean serum creatinine level, $1.5 \mathrm{mg}$ per deciliter [132.6 $\mu \mathrm{mol}$ per liter]), and elevated natriuretic peptide levels (mean N-terminal pro-brain natriuretic peptide level, $7439 \mathrm{pg}$ per milliliter). The mean ejection fraction was $35 \%$, and $27 \%$ of patients had an ejection fraction of $50 \%$ or greater. The median time from presentation to randomization was 14.6 hours, and the median duration of study-drug administration was 65.3 hours.

\section{Bolus versus Continuous Infusion}

Patients who were assigned to intravenous boluses of furosemide every 12 hours were more likely to require a dose increase at 48 hours than were those assigned to continuous intravenous infusion ( $21 \%$ vs. $11 \%, \mathrm{P}=0.01)$. There was no significant difference between these groups in the likelihood of a switch to oral diuretics at 48 hours ( $22 \%$ in the bolus group and $26 \%$ in the continuous-infusion group, $\mathrm{P}=0.44$ ). The median total dose of loop diuretics received over the course of 72 hours (in intravenous furosemide equivalents) was $592 \mathrm{mg}$ in the bolus group as compared with $480 \mathrm{mg}$ in the continuous-infusion group $(\mathrm{P}=$ 0.06) (for details, see Section 5 in the Supplementary Appendix).

There was no significant difference between the two treatment groups in the primary efficacy end point of patient-reported global assessment of symptoms (mean AUC, $4236 \pm 1440$ with boluses and $4373 \pm 1404$ with continuous infusion; $P=0.47$ ) (Fig. 1). There 
was also no significant between-group difference in the primary safety end point of the change in serum creatinine level from baseline to 72 hours (mean change in creatinine level, $0.05 \pm 0.3 \mathrm{mg}$ per deciliter $[4.4 \pm 26.5 \mu \mathrm{mol}$ per liter] with boluses and $0.07 \pm 0.3 \mathrm{mg}$ per deciliter [6.2 $\pm 26.5 \mu \mathrm{mol}$ per liter] with continuous infusion; $\mathrm{P}=0.45$ ) (Fig. 2). There was no evidence of an interaction between factorial groups (i.e., between the mode of administration and the dosing strategy) for either the primary efficacy end point $(\mathrm{P}=0.93)$ or the primary safety end point $(\mathrm{P}=0.70)$. There were also no significant between-group differences across a variety of secondary end points (Table 2). Serum creatinine and cystatin C levels were similar between the groups during the index hospitalization and at 60 days (see Section 6 in the Supplementary Appendix).

\section{Low-Dose versus High-Dose Strategy}

Patients assigned to the high-dose strategy were more likely to change to oral diuretics at 48 hours than were those assigned to the low-dose strategy ( $31 \%$ vs. $17 \%, \mathrm{P}<0.001$ ).

Conversely, patients in the low-dose group were more likely to require a $50 \%$ increase in the dose at 48 hours than were those in the high-dose group ( $24 \%$ vs. $9 \%, \mathrm{P}=0.003)$. The median total dose of loop diuretics received over the course of 72 hours (in intravenous furosemide equivalents) was $358 \mathrm{mg}$ with the low-dose strategy as compared with $773 \mathrm{mg}$ with the high-dose strategy $(\mathrm{P}<0.001)$ (for details, see Section 5 in the Supplementary Appendix).

There was a nonsignificant trend toward greater improvement in the primary efficacy end point in the high-dose group than in the low-dose group (mean AUC, $4430 \pm 1401$ vs. 4171 $\pm 1436 ; \mathrm{P}=0.06$ ) (Fig. 1). There was no significant difference between these two treatment groups in the primary safety end point (mean change in the serum creatinine level, $0.04 \pm 0.3 \mathrm{mg}$ per deciliter [3.5 $\pm 26.5 \mu \mathrm{mol}$ per liter] in the low-dose group and $0.08 \pm 0.3 \mathrm{mg}$ per deciliter [7.1 $\pm 26.5 \mu \mathrm{mol}$ per liter] in the high-dose group; $\mathrm{P}=0.21$ ) (Fig. 2).

High-dose furosemide resulted in greater net fluid loss, weight loss, and relief from dyspnea (Table 2). These potentially favorable effects of high-dose furosemide were balanced by a higher proportion of patients who met the prespecified secondary safety end point of worsening renal function (i.e., an increase in the serum creatinine level of more than $0.3 \mathrm{mg}$ per deciliter at any time during the 72 hours after randomization), which occurred in $23 \%$ of the patients in the high-dose group, as compared with $14 \%$ in the low-dose group $(\mathrm{P}=0.04)$. There were no significant differences between the two study groups in serum creatinine and cystatin $\mathrm{C}$ levels during the index hospitalization or at 60 days (see Section 6 in the Supplementary Appendix).

\section{Clinical Events}

Fewer patients in the high-dose group than in the low-dose group had a serious adverse event ( $38 \%$ vs. $50 \%, \mathrm{P}=0.03$ ). There were no differences between the bolus group and the continuous-infusion group in the proportion of patients with serious adverse events (44\% in each group, $P=0.92$ ). Individual rates of adverse events are shown in Section 4 in the Supplementary Appendix. There were more cases of ventricular tachycardia with boluses than with continuous infusion (7 vs. 4) and with the low-dose strategy than with the highdose strategy ( 7 vs. 4 ). There were similar differences with respect to cases of myocardial infarction ( 4 cases vs. 1 case with both boluses vs. continuous infusion and low-dose strategy vs. high-dose strategy). There were more cases of renal failure with continuous infusion than with boluses (11 vs. 8) and with the low-dose strategy than with the high-dose strategy (12 vs. 7). 
The median length of stay during the index hospitalization was 5 days and did not differ significantly across the treatment groups. A total of 130 patients (42\%) died, were rehospitalized, or had an emergency department visit within the 60-day follow-up period, but there was no significant difference in this composite end point between the continuousinfusion group and the bolus group (67 events and 63 events, respectively; hazard ratio with continuous infusion, $1.15 ; 95 \%$ confidence interval [CI], 0.83 to $1.60 ; \mathrm{P}=0.41$ ) or between the high-dose group and the low-dose group (63 events and 67 events, respectively; hazard ratio with high dose, $0.83 ; 95 \% \mathrm{CI}, 0.60$ to $1.16 ; \mathrm{P}=0.28$ ) (Fig. 3). The total numbers of days that patients were alive and out of the hospital were similar with the two modes of administration and the two dosing strategies (Table 2).

\section{Discussion}

Although loop diuretics are an essential component of therapy for acute decompensated heart failure, there have been few prospective data to guide decision-making regarding the use of these agents. In this trial, we found no significant differences in either patients' global assessment of symptoms or the change in the creatinine level from baseline to 72 hours when diuretic therapy was administered by means of boluses as compared with continuous infusion or with a low-dose strategy as compared with a high-dose strategy.

With respect to the comparison of bolus with continuous infusion, there was no significant difference between the treatment groups across a broad range of efficacy and safety end points. These findings are not consistent with prior, much smaller studies suggesting that continuous infusion, as compared with boluses, is associated with a lesser degree of renal dysfunction and greater diuresis. ${ }^{10-15,21}$ One possible explanation for the absence of a significant difference in outcomes between boluses and continuous infusion in our study is the use of a continuous placebo infusion in the patients assigned to boluses; this feature of the study design may have served to increase the time the patients were supine, a position that has been shown to enhance diuresis. ${ }^{22}$ In addition, it should be noted that the bolus group tended to receive a higher total dose of diuretic than did the continuous-infusion group.

With respect to the comparison of the low-dose strategy with the high-dose strategy, there was also no significant difference between the treatment groups in the primary efficacy or safety end points. The high-dose strategy was, however, associated with greater relief of dyspnea, greater fluid loss and weight loss, and fewer serious adverse events. In previous studies, greater relief of dyspnea has been associated with more favorable outcomes after discharge from the hospital. ${ }^{23}$ Although it is often assumed that dyspnea will resolve quickly with standard treatment, a recent study has suggested that moderate or severe dyspnea persists beyond the initial treatment phase in many patients with acute decompensated heart failure. ${ }^{24}$ Dyspnea was one of several secondary end points in this trial. Although the difference in the AUC measure of dyspnea between the high-dose and low-dose groups met our prespecified threshold for statistical significance, it remains possible that this was a chance finding.

Prior studies have suggested that high doses of diuretics are associated with worsening renal function, ${ }^{6}$ which has been proposed as a mechanism by which loop diuretics could lead to worse outcomes. ${ }^{5}$ Although worsening of renal function occurred more frequently with the high-dose strategy in the short term, there was no evidence at 60 days of worse clinical outcomes in the high-dose group than in the low-dose group. This observation is consistent with other recent data suggesting that transient worsening of renal function during hospitalization for heart failure may not affect the outcomes after discharge from the hospital. ${ }^{25,26}$ These findings suggest that prior observations linking high-dose diuretics with 
poor outcomes may reflect the severity of the illness rather than a harmful effect of high doses. Whether repeated episodes of transient worsening of renal function (as might occur during sequential hospitalizations) might in the long term have permanent harmful effects cannot be determined from this trial.

There are several limitations of this study. First, the patients who participated in the trial had a history of chronic heart failure and required moderate-to-high doses of loop diuretics (between 80 and $240 \mathrm{mg}$ of furosemide per day or equivalent doses of other loop diuretics) as outpatients. Our findings may not be applicable to patients with newly diagnosed heart failure or those with more modest diuretic requirements. Second, the trial was not powered to detect between-group differences in clinical events. Finally, many participants received open-label diuretic therapy during the period before randomization, and the trial also allowed for adjustments in the diuretic dosing strategy after 48 hours of the randomly assigned strategy. These adjustments may have affected the observed differences between groups at the 72-hour end points.

In conclusion, among patients with acute decompensated heart failure and moderate-to-high baseline diuretic requirements, there were no significant differences in the patients' global assessment of symptoms or in changes from baseline renal function with either bolus as compared with continuous infusion of intravenous furosemide or with a low-dose strategy as compared with a high-dose strategy.

\section{Supplementary Material}

Refer to Web version on PubMed Central for supplementary material.

\section{Acknowledgments}

The Heart Failure Clinical Research Network is supported by grants (HL084861, HL084875, HL084877, HL084889, HL084890, HL084891, HL084899, HL084904, HL084907, and HL084931) from the National Heart, Lung, and Blood Institute.

Dr. Felker reports receiving consulting fees from Amgen, Cytokinetics, Corthera, Otsuka, Novartis, and Roche Diagnostics and grant support from Amgen, Cytokinetics, Otsuka, and Roche Diagnostics; Dr. LeWinter, receiving consulting fees from Novartis, grant support from Medtronic, and lecture fees from Medtronic and Novartis; Dr. Anstrom, receiving consulting fees from Johnson \& Johnson and Pfizer; Dr. Hernandez, receiving consulting fees from Amgen and grant support from Johnson \& Johnson and serving on a clinical end points committee for Corthera; Dr. Velazquez, receiving consulting fees from Novartis and Boehringer Ingelheim, grant support from Johnson \& Johnson, and lecture fees from Novartis; Dr. Kfoury, receiving grant support from Novartis and XDx; Dr. Chen, receiving grant support from Scios, Anexon, and Nile Therapeutics, being named as a coinventor on patents for chimeric natriuretic peptides, and receiving royalties from Nile Therapeutics; and Dr. O'Connor, receiving consulting fees from Merck, GE Healthcare, Forest Pharmaceuticals, Medtronic, Novella Clinical, Medpace, Roche, Actelion Pharmaceuticals, Amgen, Trevena, and Martek Biosciences and grant support from Johnson \& Johnson.

\section{References}

1. Lloyd-Jones D, Adams R, Carnethon M, et al. Heart disease and stroke statistics - 2009 update: a report from the American Heart Association Statistics Committee and Stroke Statistics Subcommittee. Circulation. 2009; 119(3):e21-e181. [PubMed: 19075105]

2. Emerman CL, Marco TD, Costanzo MR, Peacock WF. Impact of intravenous diuretics on the outcomes of patients hospitalized with acute decompensated heart failure: insights from the ADHERE(R) Registry. J Card Fail. 2004; 10(Suppl):S116-S117.

3. Jessup M, Abraham WT, Casey DE, et al. 2009 Focused update: ACCF/AHA Guidelines for the Diagnosis and Management of Heart Failure in Adults: a report of the American College of Cardiology Foundation/American Heart Association Task Force on Practice Guidelines: developed 
in collaboration with the International Society for Heart and Lung Transplantation. Circulation. 2009; 119:1977-2016. [PubMed: 19324967]

4. Adams KF, Lindenfeld J, Arnold JM, et al. HFSA 2006 comprehensive heart failure practice guideline. J Card Fail. 2006; 12:1-119. [PubMed: 16500577]

5. Felker GM, O'Connor CM, Braunwald E. Loop diuretics in acute decompensated heart failure: necessary? Evil? A necessary evil? Circ Heart Fail. 2009; 2:56-62. [PubMed: 19750134]

6. Butler J, Forman DE, Abraham WT, et al. Relationship between heart failure treatment and development of worsening renal function among hospitalized patients. Am Heart J. 2004; 147:3318. [PubMed: 14760333]

7. Hasselblad V, Stough WG, Shah MR, et al. Relation between dose of loop diuretics and outcomes in a heart failure population: results of the ESCAPE trial. Eur J Heart Fail. 2007; 9:1064-9. [PubMed: 17719273]

8. Cooper HA, Dries DL, Davis CE, Shen YL, Domanski MJ. Diuretics and risk of arrhythmic death in patients with left ventricular dysfunction. Circulation. 1999; 100:1311-5. [PubMed: 10491376]

9. Allen LA, Turer AT, Dewald T, Stough WG, Cotter G, O'Connor CM. Continuous versus bolus dosing of furosemide for patients hospitalized for heart failure. Am J Cardiol. 2010; 105:1794-7. [PubMed: 20538132]

10. van Meyel JJ, Smits P, Dormans T, Gerlag PG, Russel FG, Gribnau FW. Continuous infusion of furosemide in the treatment of patients with congestive heart failure and diuretic resistance. $\mathrm{J}$ Intern Med. 1994; 235:329-34. [PubMed: 8151264]

11. Rudy DW, Voelker JR, Greene PK, Esparza FA, Brater DC. Loop diuretics for chronic renal insufficiency: a continuous infusion is more efficacious than bolus therapy. Ann Intern Med. 1991; 115:360-6. [PubMed: 1863026]

12. Magovern JA, Magovern GJ Jr. Diuresis in hemodynamically compromised patients: continuous furosemide infusion. Ann Thorac Surg. 1990; 50:482-4. [PubMed: 2400276]

13. Lawson DH, Gray JMB, Henry DA, Tillstone WJ. Continuous infusion of frusemide in refractory oedema. BMJ. 1978; 2:476. [PubMed: 678930]

14. Thomson MR, Nappi JM, Dunn SP, Hollis IB, Rodgers JE, Van Bakel AB. Continuous versus intermittent infusion of furosemide in acute decompensated heart failure. J Card Fail. 2010; 16:188-93. [PubMed: 20206891]

15. Dormans TPJ, Vanmeyel JJ, Gerlag PG, Tan Y, Russel FG, Smits P. Diuretic efficacy of high dose furosemide in severe heart failure: bolus injection versus continuous infusion. J Am Coll Cardiol. 1996; 28:376-82. [PubMed: 8800113]

16. Salvator DR, Rey NR, Ramos GC, Punzalan FE. Continuous infusion versus bolus injection of loop diuretics in congestive heart failure. Cochrane Database Syst Rev. 2005; 3:CD003178. [PubMed: 16034890]

17. Grant S, Aitchison T, Henderson E, et al. A comparison of the reproducibility and the sensitivity to change of visual analogue scales, Borg scales, and Likert scales in normal subjects during submaximal exercise. Chest. 1999; 116:1208-17. [PubMed: 10559077]

18. Allen LA, Metra M, Milo-Cotter O, et al. Improvements in signs and symptoms during hospitalization for acute heart failure follow different patterns and depend on the measurement scales used: an international, prospective registry to evaluate the evolution of measures of disease severity in acute heart failure (MEASURE-AHF). J Card Fail. 2008; 14:777-84. [PubMed: 18995183]

19. Costanzo MR, Guglin ME, Saltzberg MT, et al. Ultrafiltration versus intravenous diuretics for patients hospitalized for acute decompensated heart failure. J Am Coll Cardiol. 2007; 49:675-83. Erratum, J Am Coll Cardiol 2007;49:1136. [PubMed: 17291932]

20. Binanay C, Califf RM, Hasselblad V, et al. Evaluation study of congestive heart failure and pulmonary artery catheterization effectiveness: the ESCAPE trial. JAMA. 2005; 294:1625-33. [PubMed: 16204662]

21. Copeland JG, Campbell DW, Plachetka JR, Salomon NW, Larson DF. Diuresis with continuous infusion of furosemide after cardiac surgery. Am J Surg. 1983; 146:796-9. [PubMed: 6650766]

22. Abildgaard U, Aldershvile J, Ring-Larsen H, et al. Bed rest and increased diuretic treatment in chronic congestive heart failure. Eur Heart J. 1985; 6:1040-6. [PubMed: 3913604] 
23. Metra M, Cleland JG, Davison Weatherley B, et al. Dyspnoea in patients with acute heart failure: an analysis of its clinical course, determinants, and relationship to 60-day outcomes in the PROTECT pilot study. Eur J Heart Fail. 2010; 12:499-507. [PubMed: 20228387]

24. Pang PS, Konstam MA, Krasa HB, et al. Effects of tolvaptan on dyspnoea relief from the EVEREST trials. Eur Heart J. 2009; 30:2233-40. [PubMed: 19561338]

25. Aronson D, Burger AJ. The relationship between transient and persistent worsening renal function and mortality in patients with acute decompensated heart failure. J Card Fail. 2010; 16:541-7. [PubMed: 20610229]

26. Testani JM, Chen J, McCauley BD, Kimmel SE, Shannon RP. Potential effects of aggressive decongestion during the treatment of decompensated heart failure on renal function and survival. Circulation. 2010; 122:265-72. [PubMed: 20606118] 
A Bolus vs. Continuous Infusion
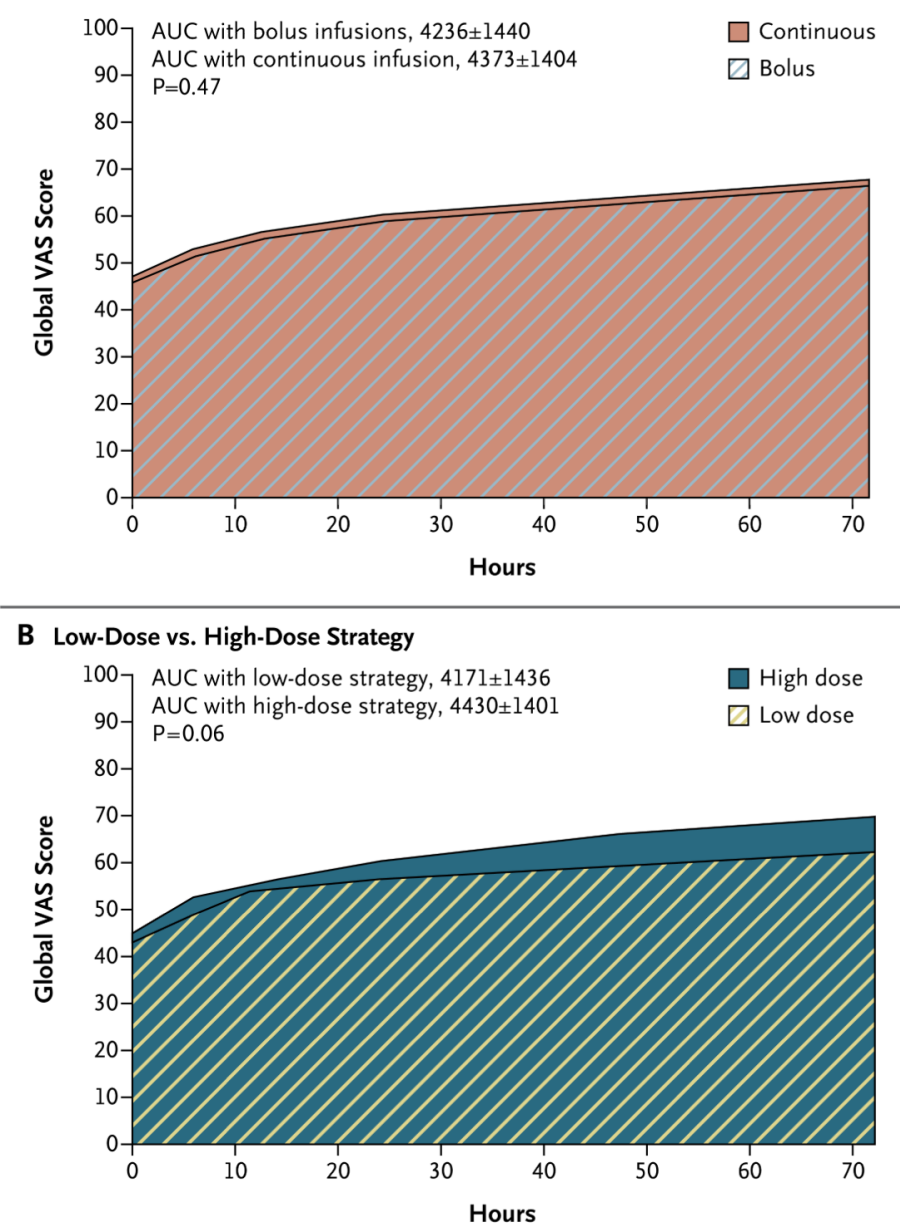

Figure 1. Patients' Global Assessment of Symptoms during the 72-Hour Study-Treatment Period Patients' global assessment of symptoms was measured with the use of a visual-analogue scale and quantified as the area under the curve (AUC) of serial assessments from baseline to 72 hours. Mean $( \pm \mathrm{SD})$ AUCs are shown for the group that received boluses every 12 hours as compared with the group that received a continuous infusion (Panel A) and for the group that received a low dose of the diuretic (equivalent to the patients' previous oral dose) as compared with the group that received a high dose (2.5 times the previous oral dose) (Panel B). Plus-minus values are means \pm SD. 


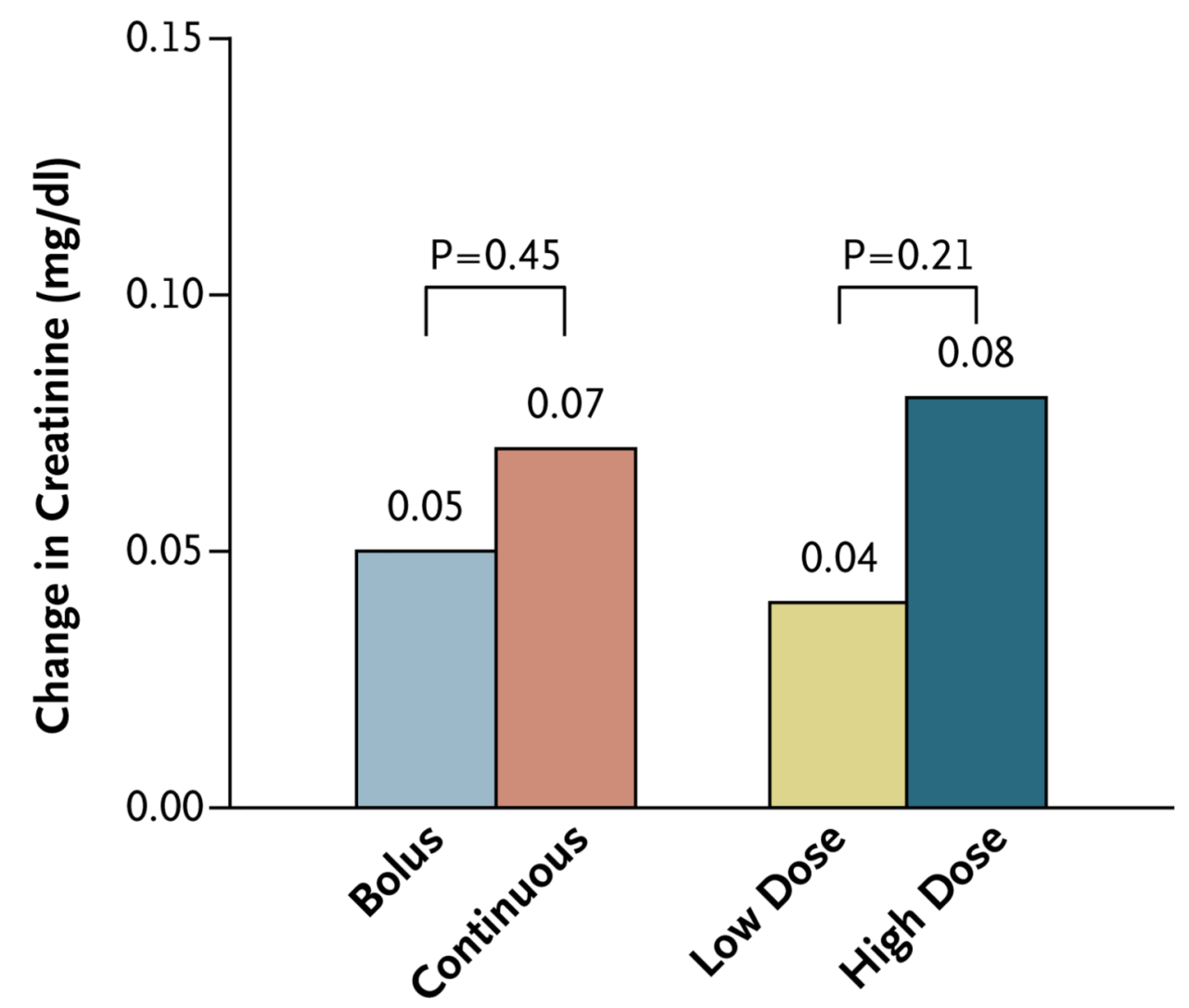

Figure 2. Mean Change in Serum Creatinine Level

The mean change in the serum creatinine level over the course of the 72-hour studytreatment period is shown for the group that received boluses every 12 hours as compared with the group that received a continuous infusion and for the group that received a low dose of the diuretic (equivalent to the patients' previous oral dose) as compared with the group that received a high dose (2.5 times the previous oral dose). To convert the values for creatinine to micromoles per liter, multiply by 88.4 . 

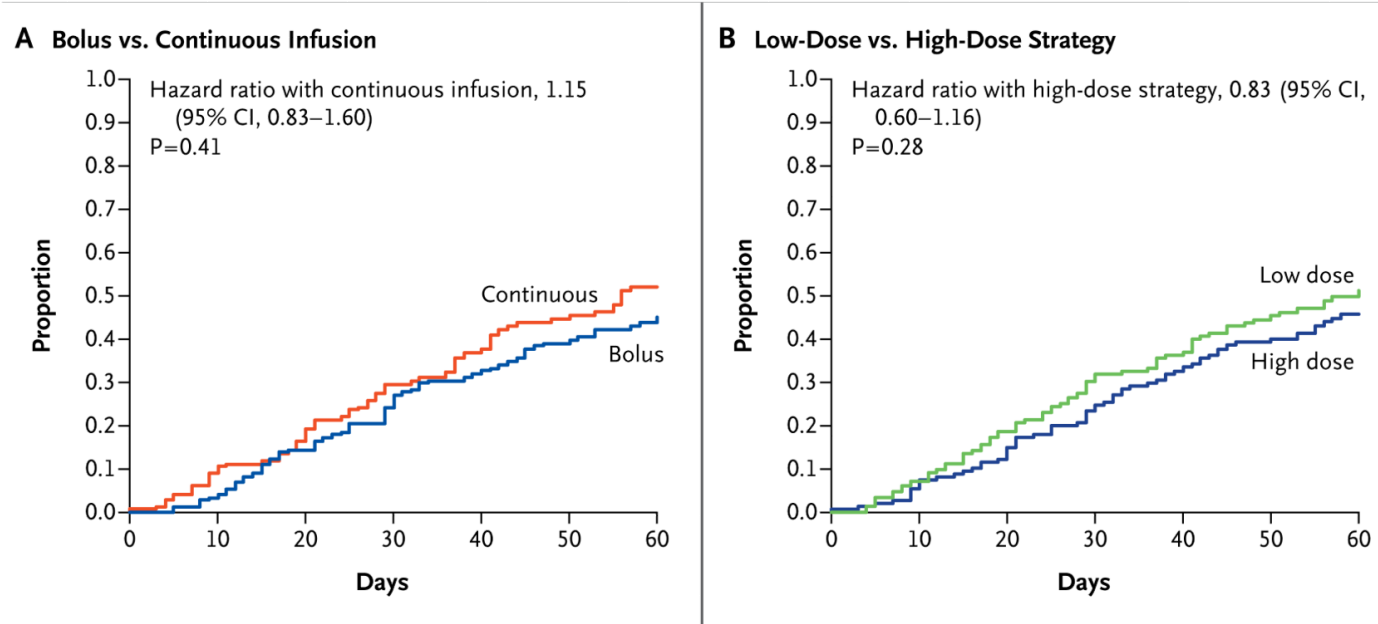

Figure 3. Kaplan-Meier Curves for the Clinical Composite End Point of Death, Rehospitalization, or Emergency Department Visit

Kaplan-Meier curves are shown for death, rehospitalization, or emergency department visit during the 60-day follow-up period in the group that received boluses every 12 hours as compared with the group that received a continuous infusion (Panel A) and in the group that received a low dose of the diuretic (equivalent to the patients' previous oral dose) as compared with the group that received a high dose (2.5 times the previous oral dose) (Panel B). 
Table 1

Baseline Characteristics of the Study Participants, According to Treatment Group. *

\begin{tabular}{|c|c|c|c|c|}
\hline Characteristic & $\begin{array}{l}\text { Bolus Every } 12 \mathrm{Hr} \\
(\mathbf{N}=156)\end{array}$ & $\begin{array}{c}\text { Continuous } \\
\text { Infusion }(\mathrm{N}=152)\end{array}$ & $\begin{array}{l}\text { Low Dose }(N= \\
151)\end{array}$ & $\begin{array}{l}\text { High Dose }(\mathrm{N} \\
\text { 157) }\end{array}$ \\
\hline Age $-\mathrm{yr}$ & $66.2 \pm 13.2$ & $65.8 \pm 14.1$ & $65.9 \pm 13.3$ & $66.2 \pm 13.9$ \\
\hline Male sex - no. $(\%)$ & $115(74)$ & $111(73)$ & $110(73)$ & $116(74)$ \\
\hline White race - no. $(\%)$ & $114(73)$ & $108(71)$ & $106(70)$ & $116(74)$ \\
\hline $\begin{array}{l}\text { Dose of oral furosemide or furosemide equivalent } \\
-\mathrm{mg} / \text { day }\end{array}$ & $134 \pm 53$ & $127 \pm 50$ & $131 \pm 52$ & $131 \pm 51$ \\
\hline Ejection fraction $(\%)$ & $35 \pm 18$ & $35 \pm 18$ & $33 \pm 17$ & $36 \pm 18$ \\
\hline $\begin{array}{l}\text { Hospitalization for heart failure within previous } 12 \\
\text { mo - no./total no. }(\%)\end{array}$ & $114 / 155(74)$ & $111 / 149(74)$ & $115 / 150(77)$ & $110 / 154(71)$ \\
\hline Ischemia as cause of heart failure - no. $(\%)$ & $91(58)$ & $85(56)$ & $88(58)$ & $88(56)$ \\
\hline History of atrial fibrillation or flutter - no. $(\%)$ & $84(54)$ & $78(51)$ & $82(54)$ & $80(51)$ \\
\hline Diabetes mellitus — no. $(\%)$ & $81(52)$ & $77(51)$ & $77(51)$ & $81(52)$ \\
\hline Implantable cardioverter-defibrillator - no. $(\%)$ & $63(40)$ & $56(37)$ & $62(41)$ & $57(36)$ \\
\hline $\mathrm{ACE}$ inhibitor or $\mathrm{ARB}-$ no. $(\%)$ & $104(67)$ & $93(61)$ & $94(62)$ & $103(66)$ \\
\hline Beta-blocker - no. $(\%)$ & $133(85)$ & $123(81)$ & $125(83)$ & $131(83)$ \\
\hline Aldosterone antagonist — no. (\%) & $42(27)$ & $44(29)$ & $43(28)$ & $43(27)$ \\
\hline Systolic blood pressure $-\mathrm{mm} \mathrm{Hg}$ & $118 \pm 19$ & $121 \pm 22$ & $120 \pm 19$ & $119 \pm 21$ \\
\hline Heart rate - beats $/ \mathrm{min}$ & $76 \pm 14$ & $80 \pm 17$ & $78 \pm 15$ & $79 \pm 17$ \\
\hline Oxygen saturation - \% & $96 \pm 3$ & $96 \pm 3$ & $96 \pm 3$ & $96 \pm 3$ \\
\hline $\begin{array}{l}\text { Jugular venous pressure } \geq 8 \mathrm{~cm} \text { of water }- \text { no./ } \\
\text { total no. }(\%)\end{array}$ & 137/151 (91) & $130 / 141(92)$ & $128 / 141(91)$ & $139 / 151(92)$ \\
\hline Orthopnea - no./total no. (\%) & $134 / 146(92)$ & $133 / 148(90)$ & $137 / 147(93)$ & $130 / 147(88)$ \\
\hline Sodium $-\mathrm{mg} / \mathrm{dl}$ & $138 \pm 4$ & $138 \pm 4$ & $138 \pm 4$ & $138 \pm 4$ \\
\hline $\mathrm{BUN}-\mathrm{mg} / \mathrm{dl}$ & $37 \pm 21$ & $38 \pm 24$ & $38 \pm 23$ & $37 \pm 22$ \\
\hline Creatinine $-\mathrm{mg} / \mathrm{dl}$ & $1.5 \pm 0.5$ & $1.5 \pm 0.5$ & $1.5 \pm 0.5$ & $1.5 \pm 0.5$ \\
\hline NT-proBNP - pg/ml & $7308 \pm 7097$ & $7570 \pm 7557$ & $8125 \pm 7624$ & $6758 \pm 6961$ \\
\hline Cystatin $\mathrm{C}-\mathrm{mg} / \mathrm{liter}$ & $1.6 \pm 0.5$ & $1.6 \pm 0.6$ & $1.6 \pm 0.5$ & $1.6 \pm 0.6$ \\
\hline
\end{tabular}

* Plus-minus values are means $\pm \mathrm{SD}$. All P values are greater than 0.05 for the comparisons of baseline characteristics across groups (bolus vs. continuous infusion and low-dose vs. high-dose strategy). To convert the values for blood urea nitrogen (BUN) to millimoles per liter, multiply by 0.357. To convert the values for creatinine to micromoles per liter, multiply by 88.4. ACE denotes angiotensin-converting enzyme, ARB angiotensin-receptor blocker, and NT-proBNP N-terminal pro-brain natriuretic peptide. 


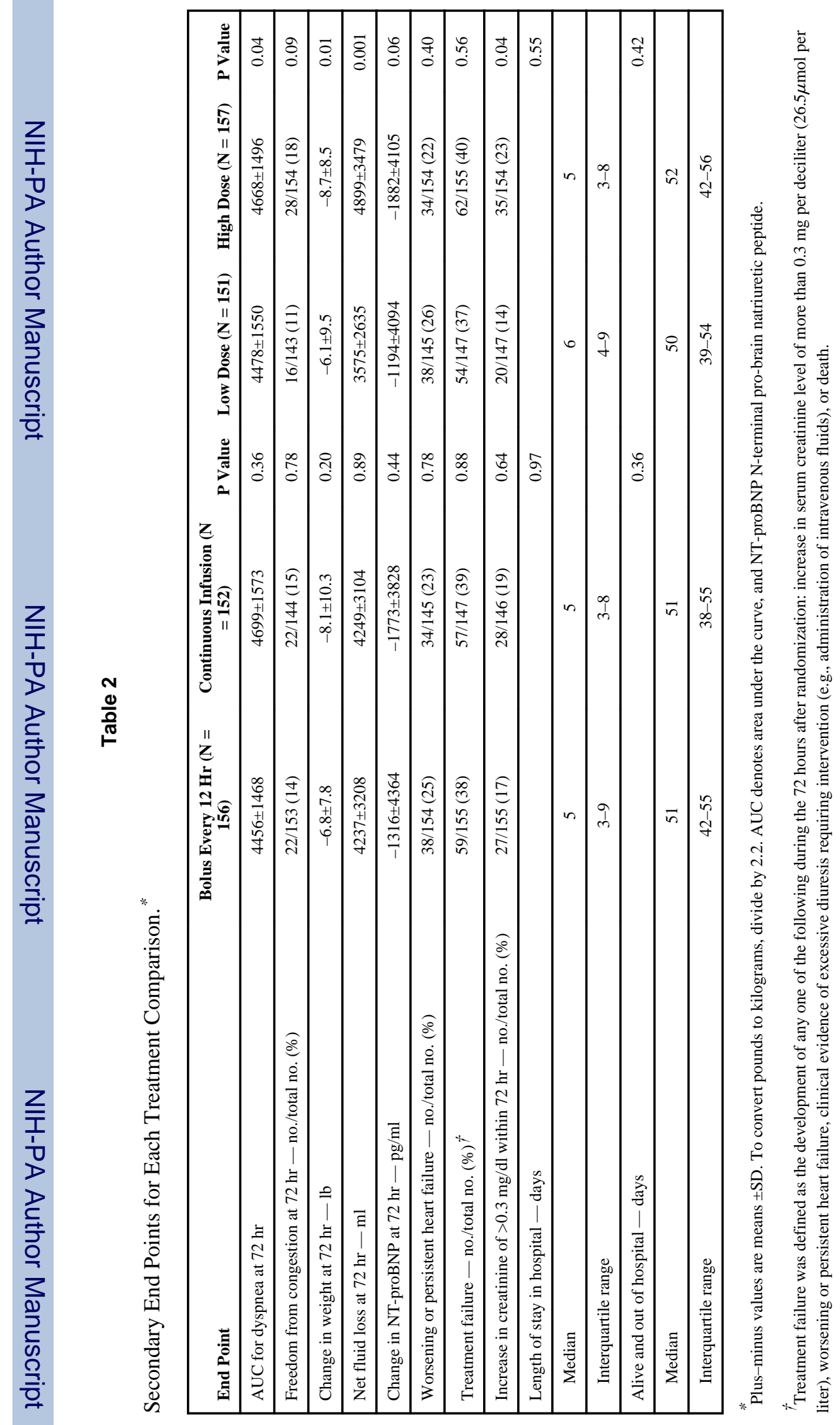

